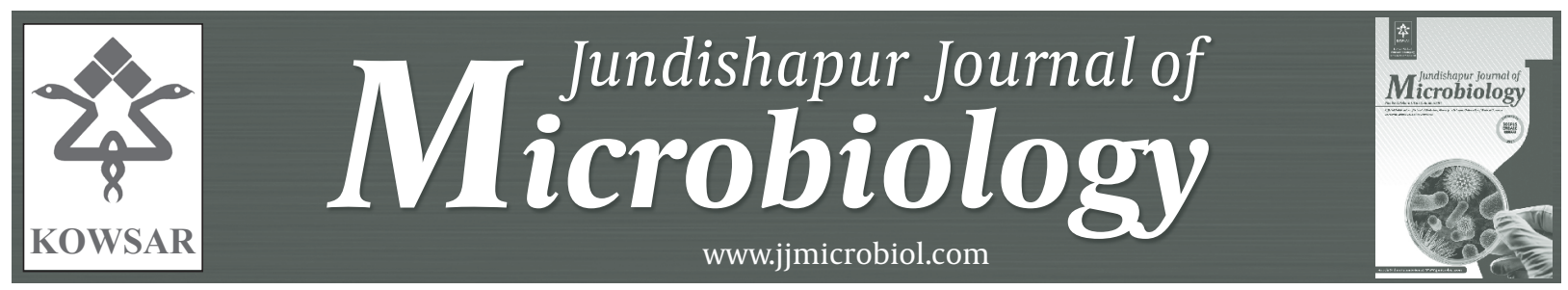

\title{
Identification of Cutaneous Leishmaniasis Agents in Four Geographical Regions of Khuzestan Province Using Nested PCR
}

\author{
Sharif Maraghi ${ }^{1,{ }^{*}}$, Omid Mardanshah ${ }^{1}$, Abdollah Rafiei ${ }^{2}$, Alireza Samarbafzadeh ${ }^{3}$, Babak \\ Vazirianzadeh $^{4}$ \\ ${ }^{1}$ Department of Parasitology and Mycology, Arvand International Division, Infectious and Tropical Diseases Research Center, Thalassemia and Haemoglo- \\ binopathy Research Center, Ahvaz, IR Iran \\ ${ }^{2}$ Department of Parasitology, School of Medicine, Infectious and Tropical Diseases Research Center, Ahvaz, IR Iran \\ ${ }^{3}$ Department of Virology, School of Medicine, Ahvaz, IR Iran \\ ${ }^{4}$ Department of Entomology, School of Health, Infectious and Tropical Diseases Research Center, Ahvaz Jundishapur University of Medical Sciences, \\ Ahvaz, IR Iran \\ ${ }^{*}$ Corresponding author: Sharif Maraghi, Department of Parasitology and Mycology, Arvand international division, Infectious and Tropical Diseases Re- \\ search Center, Thalassemia and Haemoglobinopathy, Research Center Ahvaz, IR Iran. Tel:+98-6113330678, E-mail: maraghis@gmail.com.
}

\begin{abstract}
A B S T R A C T
Background: Leishmania tropica and Leishmania major are the main causes of cutaneous leishmaniasis in endemic regions of Iran.

Objectives: The aim of this study was the identification of cutaneous leishmaniasis agents in Khuzestan province located southwest of Iran. Patients and Methods: 146 samples were collected from the lesions of 146 individuals including $67(59.59 \%)$ male and 59 ( $40.41 \%$ ) female with cutaneous leishmaniasis. The samples were then delivered to Iran-Zamin diagnostic laboratory and smeared on slides, stained with Wright's eosin methylene blue stain and examined microscopically and graded from $1+$ to $4+$. DNA was extracted from the slides and the identification of cutaneous leishmaniasis agents was performed using Nested PCR with the primers of CSB1XR, CSB2XF, LIR and $13 Z$.

Results: 138 (94.5\%) out of 146 cases of four regions were L. major and 8 (5.5\%) were L. tropica. 57.97\% of L. major cases were male and $42.03 \%$ were female. $87.5 \%$ of $L$. tropica were male and $12.5 \%$ were female. The maximum number of $L$. tropica cases was found in the northern region (8.16\%) and the minimum was found in the western region (3.22\%). 96.78\% of L. major cases belonged to the western region of Khuzestan.

Conclusions: L. major is the main species responsible for cutaneous leishmsniasis in four geographical regions of Khuzestan province southwestern of Iran and Nested PCR can be used for diagnosis and Leishmania species identification.
\end{abstract}

Keywords: Cutaneous leishmaniasis; Leishmania Major; Leishmania Tropica, PCR; Iran

Copyright ( (2013, Ahvaz Jundishapur University of Medical Sciences; Published by Kowsar Corp.

Article type: Research Article; Received: 30 Apr 2012; Revised: 20 Jun 2012; Accepted: 08 Jul 2012; Epub: 01 Jun 2013; Ppub: Jun 2013

Implication for health policy/practice/research/medical education:

The results of this study indicated that the main causative agent of cutaneous leismaniasis in Khuzestan province is L. major and nested PCR is the best method for diagnosis and identification of causative agents.

Please cite this paper as:

Maraghi S, Mardanshah O, Rafiei A, Samarbafzadeh A, Vazirianzadeh B. Identification of Cutaneous Leishmaniasis Agents in Four Geographical Regions of Khuzestan Province Using Nested PCR. Jundishapur J Microbiol. 2013;6(4):e4866. DOI: 10.5812/jjm.4866

Copyright @ 2013, Ahvaz Jundishapur University of Medical Sciences; Published by Kowsar Corp.

This is an Open Access article distributed under the terms of the Creative Commons Attribution License (http://creativecommons.org/licenses/by/3.0), which permits unrestricted use, distribution, and reproduction in any medium, provided the original work is properly cited. 


\section{Background}

Leishmaniases are known as a group of globally widespread parasitic diseases caused by different species of Leishmania, which is capable of infecting a wide variety of mammals. Currently, 22 species of Leishmania are pathogen for human, infection when exposed to the natural transmission cycle $(1,2)$. Leishmaniases have been considered tropical afflictions that together constitute one of the entities on the World Health Organization/Tropical six Disease Research (WHO/TDR) list of most important diseases $(3,4)$. The disease is endemic in 88 countries of 5 continents with a total of 350 -million people at risk and 12 million cases. Among the 88 endemic countries, 22 are in the New World and 66 in the Old World with an estimated incidence of 1-1.5 million cases of cutaneous leishmaniasis (CL) and 500, 000 cases of visceral leishmaniasis (VL) (3).

Direct microscopic examinations of stained smears or tissue samples usually used for CL diagnosis, is rapid and easy to use for diagnosis of cutaneous leishmaniasis. But studies in endemic regions revealed that a considerable number of clinically diagnosed cannot be confirmed by microscopic examinations and that methods with more sensitivity and specificity should be implemented in CL diagnosis (5). Polymerase chain reaction (PCR)-based methods have provided the capability of diagnosis and also identification of Leishmania species. Different species may have the criteria for the treatment phase (6). The main biological samples used for diagnosis and identification of CL species by PCR are dermal scrapings or biop$\operatorname{sies}(7,8)$.

\section{Objectives}

The purpose of this study was the identification of cutaneous leishmaniasis causative agents in four geographical regions in Khuzestan province, Iran.

\section{Patients and Methods}

\subsection{Samples}

146 smears were prepared from the margins of patients lesions referred to Iran- Zamin diagnostic laboratory from four regions of Khuzestan province (northern, southern, eastern and western regions) ( Figure 1 ). The smears were dried and stained with Wright's eosin-meth- yleneblue and examined microscopically and graded as $1+, 2+, 3+$ and $4+$, according to number of amastigotes in each high power field of microscope (9).

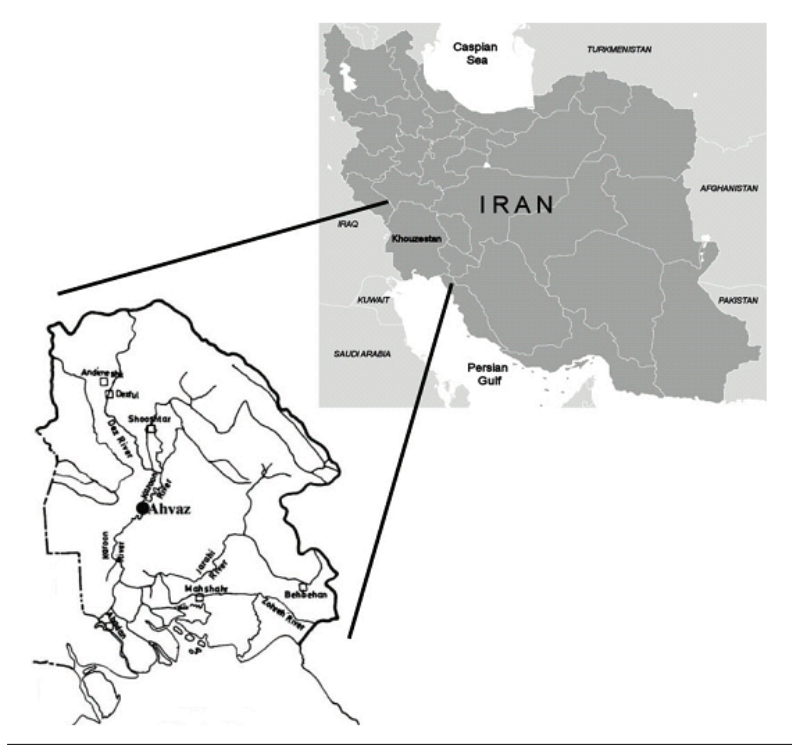

Figure 1. Map of Iran, Showing the Location of Khuzestan Province

\subsection{DNA Extraction}

Amastigotes on the stained slides were scraped and DNA extraction was performed according to the protocol of commercial kit (Bioneer, South Korea).

\subsection{Nested-PCR}

External primers CSB1XR (ATTTTTCG/CGA/TTTT/CGCAGAA CG) and CSB2XF(C/GA/GTA /GCAGAAAC/TCCCGTTCA) and the internal primers, 13Z (ACTGGGGGTTGGTGTAAAATAG) and LiR (TCGCAGAACGCCCCT) were used to amplify the minicircle variable kDNA. Two Leishmania species produced the amplified fragments of about 750 $\mathrm{bp}$ for L. tropica $\mathrm{f}$ and $560 \mathrm{bp}$ for L. major (10). Amplification reactions visualized in 1.5 \% Agarose Gel Electrophoresis, using a 100 bp DNA ladder (11) ( Figure 2 ).

\section{Results}

87 (59.59\%) out of 146 cases were male and 59 (40.41\%) were female. Patients' ages ranged from 10 to 41 years. 138 (94.52\%) cases were infected with L.major and 8 (5.48\%) patients were infected with L.tropica.Thefrequency of cu- 
taneous leishmaniasis in four regions of Khuzestan province is shown in Table 1.

Figure 2. Electrophoresis of Nested

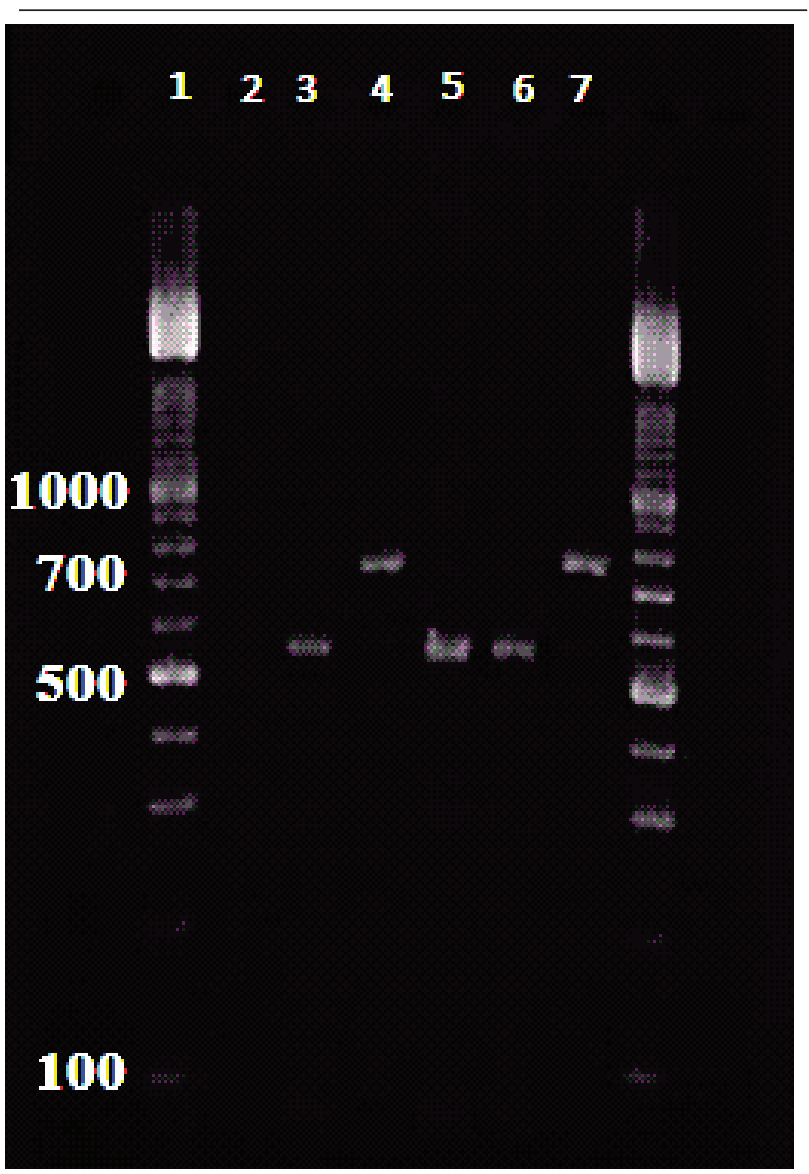

PCR products. Lane 1: Ladder marker; lane 2: negative control, 3. L. major (positive control); 4. L. tropica (positive control). Lanes 5 and 6. L. major. L ane 7. L. tropica. $750 \mathrm{bp}$ and $560 \mathrm{bp}$ bands represent $L$. tropica and $L$. major respectively.

Table 1. Geographical Distribution of $L$.tropica and L. major in Patients With Cutaneous Leishmaniasis From Four Regions in Khuzestan Province

\begin{tabular}{lll}
\hline $\begin{array}{l}\text { Geographical Re- } \\
\text { gions }\end{array}$ & L. major & L.tropica \\
\hline No. $(\%)$ & No. $(\%)$ \\
\hline South Khuzestan & $23(95.84)$ & $4(8.16)$ \\
\hline West Khuzestan & $30(96.78)$ & $1(4.16)$ \\
\hline East Khuzestan & $40(95.24)$ & $2(4.76)$ \\
\hline
\end{tabular}

\section{Discussion}

Laboratory diagnosis of cutaneous leishmaniasis is accomplished by direct demonstration of the parasite by microscopic examination of stained specimens, by isolation of the cultured parasite in the appropriate tissue. Lately, PCR-based methods have proven to be highly sensitive and specific compared to the standard methods and are considered exceedingly valuable for diagnosis (7, $12,13)$. In endemic areas where more than one Leishmania Species are present, some diagnostic tools are required for the detection of parasites directly in the samples and the distinction of all relevant Leishmania species(14).

Identification of the Leishmania type is important, because different species may require distinct treatment regimens (15). Furthermore, such data are also valuable in epidemiologic studies where the distribution of Leishmania species in human and animal hosts, is a prerequisite for designing appropriate control measures (14, 16). In this study, L.major was the main type of cutaneous leishmaniasis in four geographical regions of Khuzestan province. $94.52 \%$ of the cases were L.major and $4.41 \%$ were L.tropica. This finding is consistent with the findings of the study conducted by Maraghi et al. in Shush (17) and Ghasemian et al. in Ahvaz (18). Saki et al. indicated that $L$. major was the main causative agent of cutaneous leishmaniasis (96.6\%) using RFLP-PCR and Mini-exon genotyping (19).

The majority of $L$. major cases belonged to the northern Khuzestan while the maximum number of L.tropica cases were found in the western region of the province. $57.97 \%$ of $L$. major cases were found in males and $42.03 \%$ in females, whereas the gender-based frequencies for $L$. tropica were $87.5 \%$ and $12.5 \%$ respectively. The duration of lesions in L. tropica was longer than L. major. It is concluded that $L$.major is the main causative agent of cutaneous leishmaniasis in Khuzestan province and Nested-PCR is an appropriate method for diagnosis and identification of Leishmania species.

\section{Acknowledgements}

This study is adapted from the Ph.D thesis of Mr. Omid Mardanshah conducted under the financial support of Ahvaz Jundishapur University of Medical Sciences (AJUMS).

\section{Financial Disclosure}

None declared.

\section{Funding/Support}

None declared.

\section{Authors' Contribution}

None declared.

\section{References}

1. Ashford RW. The leishmaniases as emerging and reemerging 
zoonoses. Int J Parasitol. 2000;30(12-13):1269-81.

2. Silveira FT, Ishikawa EA, De Souza AA, Lainson R. An outbreak of cutaneous leishmaniasis among soldiers in Belem, Para State, Brazil, caused by Leishmania (Viannia) lindenbergi n. sp. A new leishmanial parasite of man in the Amazon region. Parasite. 2002;9(1):43-50.

3. Desjeux P. The increase in risk factors for leishmaniasis worldwide. Trans R Soc Trop Med Hyg. 2001;95(3):239-43.

4. Herwaldt BL. Leishmaniasis. Lancet. 1999;354(9185):1191-9.

5. Faber WR, Oskam L, van Gool T, Kroon NC, Knegt-Junk KJ, Hofwegen $\mathrm{H}$, et al. Value of diagnostic techniques for cutaneous leishmaniasis. JAm Acad Dermatol. 2003;49(1):70-4.

6. Blum J, Desjeux P, Schwartz E, Beck B, Hatz C. Treatment of cutaneous leishmaniasis among travellers. I Antimicrob Chemother 2004;53(2):158-66.

7. Chargui N, Bastien P, Kallel K, Haouas N, Akrout FM, Masmoudi A et al. Usefulness of PCR in the diagnosis of cutaneous leishmaniasis in Tunisia. Trans R Soc Trop Med Hyg. 2005;99(10):762-8.

8. Safaei A, Motazedian MH, Vasei M. Polymerase chain reaction for diagnosis of cutaneous leishmaniasis in histologically positive, suspicious and negative skin biopsies. Dermatology. 2002;205(1):18-24

9. Noyes HA, Reyburn H, Bailey JW, Smith D. A nested-PCR-based schizodeme method for identifying Leishmania kinetoplast minicircle classes directly from clinical samples and its application to the study of the epidemiology of Leishmania tropica in Pakistan. J Clin Microbiol.1998;36(10):2877-81.

10. Karamian M, Motazedian MH, Fakhar M, Pakshir K, Jowkar F, Rezanezhad H. Atypical presentation of Old-World cutaneous leishmaniasis, diagnosis and species identification by PCR.J Eur Acad Dermatol Venereol. 2008;22(8):958-62.

11. Motazedian MH, Mehrabani D, Oryan A, Asgari Q, Karamian M,
Kalantari M. Life cycle of cutaneous leishmaniasis in Larestan, southern Iran. Iran J Clin Infect Dis. 2006;1(3)

12. Lachaud L, Dereure J, Chabbert E, Reynes J, Mauboussin JM, Ozio E, et al. Optimized PCR using patient blood samples for diagnosis and follow-up of visceral Leishmaniasis, with special reference to AIDS patients. JClin Microbiol. 2000;38(1):236-40.

13. Mauricio IL, Stothard JR, Miles MA. Leishmania donovani complex: genotyping with the ribosomal internal transcribed space and the mini-exon. Parasitology. 2004;128(Pt 3):263-7.

14. Schonian G, Nasereddin A, Dinse N, Schweynoch C, Schallig HD Presber W, et al. PCR diagnosis and characterization of Leishmania in local and imported clinical samples. Diagn Microbiol Infect Dis. 2003;47(1):349-58.

15. Leishmaniasis disease information. Available from: http://www. WHO.int/tdr/diseases/leish.2002.

16. el Tai NO, Osman OF, el Fari M, Presber W, Schonian G. Genetic heterogeneity of ribosomal internal transcribed spacer in clinical samples of Leishmania donovani spotted on filter paper as revealed by single-strand conformation polymorphisms and sequencing. Trans R Soc Trop Med Hyg. 2000;94(5):575-9.

17. Maraghi S, Samarbafzadeh AR, Sarlak AA, Ghasemian M, Vazirianzadeh B. Identification of Cutaneous Leishmaniasis Agents by Nested Po-lymerase Chain Reaction (Nested-PCR) in Shush City, Khuzestan Province, Iran. Iran J Parasitol. 2007;2(3).

18. Ghasemian M, Maraghi S, Samarbafzadeh AR, Jelowdar A, Kalan tari M. The PCR-based detection and identification of the parasites causing human cutaneous leishmaniasis in the Iranian city of Ahvaz. Ann Trop Med Parasitol. 2011;105(3):209-15.

19. Saki J, Meamar A, Oormazdi H, Akhlaghi L, Maraghi S, Mohebali M, et al. Mini-exon genotyping of leishmania species in khuzestan province, southwest iran. Iran J Parasitol. 2010;5(1):25-34 